\title{
Asociación entre compromiso religioso y presentar actitudes negativas hacia hombres homosexuales, en un grupo de estudiantes de medicina peruanos Association between religious commitment and having negative attitudes toward homosexual men, in a group of Peruvian medical students
}

Correspondencia Crislee Elizabeth Lopez crisleelopez@gmail.com

Recibido: 06/12/2016 Arbitrado por pares Aprobado: 21/02/2017

Citar como:Lopez CE, TaypeRondán A. Asociación entre compromiso religioso y presentar actitudes negativas hacia hombres homosexuales, en un grupo de estudiantes de medicina peruanos Acta Med. Peru. 2017;34(1):33-40
Crislee E. Lopez ${ }^{1,2, a}$, Alvaro Taype-Rondán ${ }^{3}$

Centro de Investigación y Estudios Médicos - CIEM. Arequipa, Perú.

2 Facultad de Medicina Humana de la Universidad Católica de Santa María. Arequipa, Perú.

3 CRONICAS Centro de Excelencia en Enfermedades Crónicas, Universidad Peruana Cayetano Heredia. Lima, Perú.

a Estudiante de Medicina Humana

\section{RESUMEN}

Objetivo: evaluar la asociación entre compromiso religioso y presentar actitudes negativas hacia hombres homosexuales, en un grupo de estudiantes de medicina peruanos. Materiales y métodos: estudio transversal analítico realizado en estudiantes que asistieron a una asamblea estudiantil de la International Federation of Medical Students Associations en Perú. La presencia de actitudes negativas hacia hombres homosexuales se evaluó usando una dimensión de la AttitudesToward Lesbians and Gay Men Scale. El compromiso religioso se evaluó con la pregunta: ¿te consideras una persona religiosa? Los factores asociados a presentar actitudes negativas hacia hombres homosexuales fueron evaluados usando razones de prevalencia (RP) y sus intervalos de confianza al 95\% (IC 95\%) calculados con regresiones de Poisson con varianza robusta. Resultados: se analizaron datos de 117 participantes (62,4\% fueron mujeres, edad promedio: $20,9 \pm 1,6$ años). Se encontró una asociación entre el compromiso religioso y presentar actitudes negativas hacia hombres homosexuales (RP: 1,89 ; IC 95\%: 1,07-3,37). Otros factores asociados fueron ser mujer (RP: 0,60; IC 95\%: 0,38-0,95) y tener algún amigo o familiar homosexual (RP: 0,38; IC 95\%: $0,24-0,61$ ). Conclusión: en la población estudiantil analizadad, el compromiso religioso se asoció con la presencia de actitudes negativas hacia hombres homosexuales. Esto sugiere la necesidad de realizar intervenciones que concilien actitudes positivas frente a la homosexualidad con la religiosidad en estudiantes de medicina.

Palabras clave: Homofobia; Homosexualidad masculina; estudiantes de medicina; Perú (fuente: DECS/Bireme). 


\begin{abstract}
Objective: To assess the association between religious commitment and having a negative attitude towards homosexual men in a group of Peruvian medical students. Materials and methods: This is a cross-sectional analytical study performed in a group of medical students who assisted to a meeting held by the International Federation of Medical Students Associations in Peru. The presence of negative attitudes towards homosexual men was assessed using a dimension of the Attitudes Towards Lesbians and Gay Men Scale. Religious commitment was assessed with the question "Do you consider yourself a religious person?" Factors associated to having negative attitudes towards homosexual men were assessed using prevalence ratios (PR) and their $95 \%$ confidence intervals $(95 \% \mathrm{Cl}$ ) calculated with Poisson regressions with robust variance. Results: Data from 117 participants was analyzed (62.4\% were female, mean age: $20.9 \pm 1.6$ years). We found an association between religious commitment and having negative attitudes towards homosexual men (PR: $1.89,95 \% \mathrm{Cl}: 1.07-3.37$ ). Other associated factors were: being a woman (PR: $0.60,95 \% \mathrm{Cl}: 0.38-0.95$ ) and having a homosexual friend or relative (PR: $0.38,95 \% \mathrm{Cl}: 0.24-0.61$ ). Conclusion: In surveyed students, religious commitment was associated with having negative attitudes towards homosexual men. This suggests that there is a need for interventions that reconcile positive attitudes towards homosexuality with religiosity in medical students.
\end{abstract}

Keywords: Homophobia; Homosexuality male; medical students; Peru (source: MeSH/NLM).

\section{INTRODUCCIÓN}

Se define por homosexualidad a la atracción sexual entre personas del mismo género ${ }^{[1]}$. Anteriormente, esta orientación sexual era catalogada como un trastorno mental para el cual se utilizaban tratamientos como el psicoanálisis, la modificación de conducta y el recondicionamiento ${ }^{[2]}$. En el año de 1973 la American Psychiatric Association concluyó que no se trataba de un trastorno mental por lo que la retiró del Diagnostic and Statistical Manual of Mental Disorders ${ }^{[3]}$.

La homofobia, definida como la aversión o intolerancia irracional a los homosexuales ${ }^{[4]}$; se puede medir con instrumentos que evalúen las actitudes negativas hacia la comunidad de lesbianas, gais, transexuales y bisexuales (LGTB). La homofobia es un problema frecuente ${ }^{[5-8]}$ que se ha asociado a ciertas características de los agresores tales como ser de sexo masculino, tener menor edad, tener menor grado de instrucción, vivir en una cultura machista, habitar en ubicaciones geográficas poco accesibles, ciudades con escaso flujo turístico y sin puertos, tener menor familiaridad o amistad con personas homosexuales y tener un mayor nivel de compromiso religioso ${ }^{[9-11]}$.

Resulta importante estudiar la discriminación hacia los homosexuales por parte de estudiantes y profesionales de la salud, debido a que estos profesionales deberían ser los indicados para prestar cuidado y brindar información sobre la homosexualidad, tanto a sus pacientes homosexuales como a la población en general. Sin embargo, cuando las actitudes de estos profesionales son negativas, resulta imposible establecer una adecuada relación médico-paciente, lo cual influye negativamente en la prestación de atención en salud hacia esta población, como se ha descrito en estudios previos ${ }^{[12,13]}$.

Una revisión sistemática sobre homofobia en estudiantes de medicina encontró estudios realizados en Estados Unidos, Australia, Inglaterra, Inglaterra, Australia y China en los que la prevalencia de homofobia varió del $10 \%$ al $25 \%{ }^{[14]}$. Otro estudio desarrollado en estudiantes de medicina de la India, utilizando la encuesta AHO (en inglés: Attitudes towards Homosexuals Questionnaire), halló que los estudiantes varones presentaban un mayor nivel de homofobia en comparación con las estudiantes

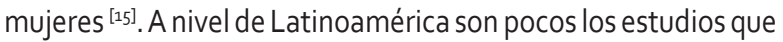
han evaluado las actitudes negativas frente a homosexuales en estudiantes de ciencias de la salud; un estudio desarrollado en México que incluyó a 231 estudiantes de psicología y medicina reportó homofobia en un $4 \%{ }^{[16]}$.

En el Perú, un estudio que incluyó a 66 estudiantes de Medicina Humana de sexto año de una universidad de Lambayeque que indagó acerca de la actitud respecto a conflictos socio-sexuales aún vigentes en la sociedad peruana, encontró que tanto el $53,1 \%$ de los varones como el $52,9 \%$ de las mujeres estaban en desacuerdo o totalmente en desacuerdo con el matrimonio civil homosexual, y que el $73,5 \%$ de los varones y el $82,4 \%$ de las mujeres estuvieron en desacuerdo o totalmente en desacuerdo con la adopción de niños por parte de parejas homosexuales ${ }^{[17]}$.

Una pregunta importante y a la vez delicada en la educación médica es cómo afecta la religión del estudiante en las actitudes que tienen frente a los homosexuales. El presente estudio se realizó con el objetivo de evaluar la asociación entre el compromiso religioso y la presencia de actitudes negativas hacia hombres homosexuales, en estudiantes de medicina humana miembros de la International Federation of Medical Students Associations en Perú (IFMSA-Perú).

\section{MATERIALES Y MÉTODOS}

\section{Diseño de estudio y población}

Se realizó un estudio transversal analítico en estudiantes de medicina humana que asistieron a la XXXI Asamblea General Ordinaria (AGO) de la IFMSA realizado durante el mes de julio del año 2015. 
Tabla 1. Características sociodemográficas de los participantes $(n=117)$

\begin{tabular}{|c|c|}
\hline Variables & $\mathbf{N}(\%)$ \\
\hline Edad (años)* & $20,9 \pm 1,6$ \\
\hline Sexo femenino & $73(62,4)$ \\
\hline \multicolumn{2}{|l|}{ Tipo de Universidad } \\
\hline Universidad privada & $103(88,1)$ \\
\hline Universidad pública & $14(11,9)$ \\
\hline \multicolumn{2}{|l|}{ Año en curso } \\
\hline 1er-3er Año & $63(53,8)$ \\
\hline 4to-7mo Año & $54(46,1)$ \\
\hline \multicolumn{2}{|l|}{ Años activo en IFMSA } \\
\hline$<1$ año & $80(68,4)$ \\
\hline >1año & $37(31,6)$ \\
\hline \multicolumn{2}{|l|}{ Religión } \\
\hline Católico & $93(79,5)$ \\
\hline Ateo & $6(5,1)$ \\
\hline Otros & $18(15,4)$ \\
\hline \multicolumn{2}{|l|}{ Familiar o amigo gay/lesbiana } \\
\hline Sí tiene & $78(66,7)$ \\
\hline No tiene & $19(16,2)$ \\
\hline No sabe & $20(17,1)$ \\
\hline \multicolumn{2}{|l|}{ Nivel de compromiso religioso } \\
\hline Poco/ muy poco religioso(a) & $42(35,9)$ \\
\hline Algo/ muy religioso(a) & $75(64,1)$ \\
\hline
\end{tabular}

* Se indica la media y desviación estándar. IFMSA: International Federation of Medical Students Associations.

\section{IFMSA}

La IFMSA es una federación que reúne a asociaciones locales de estudiantes de medicina de humana más de 115 países con el objetivo de empoderar y capacitar a estudiantes de medicina para que tomen parte activa del desarrollo de su comunidad. IFMSA está conformado por seis comités: Standing Committee on Public Health (SCOPH), Standing Committee on Sexual and Reproductive Health including HIV/AIDS (SCORA), Standing Committee on Human Rights and Peace (SCORP), Standing Committee on Medical Education (SCOME), Standing Committee on Professional Exchange (SCOPE) y Standing Committee on Research exchange (SCORE). SCORA, SCORP y SCOPH, entre otras funciones, realizan actividades contra la estigmatización de los homosexuales como campañas de protección a los derechos de la comunidad LGTB, y brindan información y promueven capacitaciones sobre en estos tópicos a sus miembros. IFMSA-Perú, fundada en el año 2000, cuenta en la actualidad con 21 asociaciones de estudiantes de medicina, ocho se encuentran en la ciudad de Lima.

\section{Procedimientos}

Del 23 al 26 de julio del 2015 se realizó la XXXI AGO de IFMSAPerú en la Universidad Católica de Santa María (Arequipa, Perú) con el objetivo de exponer las actividades regulares de la Asociación. En dicha reunión asistieron representantes de 15 de las 21 asociaciones de estudiantes afiliadas.

Al iniciar la AGO, se expuso el objetivo del estudio y se entregó las encuestas junto al material asignado para el congreso. Además, se entregó una hoja de consentimiento informado que contenía los objetivos del estudio y resaltaba el carácter voluntario y anónimo de la encuesta. La autora del estudio estuvo presente en el momento del llenado de las encuestas con el fin de absolver posibles dudas de los participantes. El tiempo dado para el rellenado de las encuestas fue de 20 minutos.

\section{Actitudes frente a homosexuales}

Se aplicó parte de la escala Attitudes Toward Lesbians and Gay Men Scale (ATLG) abreviada para evaluar la actitud de los estudiantes de medicina frente a los homosexuales ${ }^{[16]}$. Esta escala tiene una consistencia interna alta (alfa de Cronbach $=0,85$ ) y ya ha sido usada en México ${ }^{[18]}$, Colombia ${ }^{[19]}$ y Chile ${ }^{[20,21]}$. Originalmente, se divide en dos partes de 10 ítems cada una: la primera evalúa la actitud frente a los hombres homosexuales y la segunda frente a las lesbianas. Cada ítem cuenta con cinco opciones de respuesta en escala de Likert que tienen los puntajes 1, 3, 5, 7, y 9 . Para calcular el puntaje final se suman los puntajes de cada pregunta (teniendo en cuenta que en algunos ítems el puntaje se invierte), de manera que un mayor puntaje final equivale a actitudes más negativas frente a esta orientación sexual ${ }^{[20]}$.

Para este estudio se utilizaron solo los 10 ítems referidos a la actitud frente a la homosexualidad en hombres de la escala ATLG, ya que la intención era realizar una encuesta breve y debido a que el principal problema reportado en Latinoamérica son las actitudes negativas frente a los hombres homosexuales ${ }^{[22-26]}$. La escala ATLG dedicada a evaluar la actitud frente a hombres homosexuales incluye seis ítems (los ítems 2, 3, 4, 6, 8 y 9) que reflejan las actitudes negativas a la conducta homosexual, y los cuatro restantes (los ítems 1, 5, 7 y 10) las actitudes positivas. Debido a que no se cuenta con un punto de corte establecido para esta subescala, el puntaje obtenido se categorizó para poder comparar el tercil superior (mayor a 40 puntos) con los dos terciles inferiores (menor o igual a 40 puntos).

\section{Nivel de compromiso religioso}

El nivel de compromiso religioso, definido como el nivel en el cual las creencias religiosas y la doctrina influencian en las acciones del individuo y actitudes en su vida, fue evaluado mediante la pregunta "¿te consideras una persona religiosa?", que ha sido usada en estudios previos realizados en médicos ${ }^{[22,23]}$. Esta pregunta tuvo cuatro posibles alternativas: muy poco religiosa, poco religiosa, algo religiosa, y muy religiosa. Para el análisis esta variable se dicotomizó en dos categorías: 1) poco o muy poco religioso(a); y 2) algo o muy religioso(a). 
Tabla 2. Factores asociados a tener un puntaje mayor a 40 en la escala ATLG, en un grupo de estudiantes de medicina peruanos.

\begin{tabular}{|c|c|c|c|c|c|c|}
\hline Característica & $\begin{array}{c}\text { ATLG } \leq 40 \\
(\mathrm{~N}=78)\end{array}$ & $\begin{array}{c}\text { ATLG }>40 \\
(\mathrm{~N}=39)\end{array}$ & $\begin{array}{c}\text { Crudo } \\
\text { RP (IC95\%) }\end{array}$ & $\begin{array}{c}\text { Valor } \\
p\end{array}$ & $\begin{array}{c}\text { Ajustado* } \\
\text { RP (IC95\%) }\end{array}$ & $\begin{array}{c}\text { Valor } \\
p\end{array}$ \\
\hline \multicolumn{7}{|l|}{ Sexo } \\
\hline Sexo masculino & $25(56,8)$ & $19(43,2)$ & Referencia & & Referencia & \\
\hline Sexo femenino & $53(72,6)$ & $20(27,4)$ & $0,63(0,38-1,05)$ & 0,078 & $0,60(0,38-0,95)$ & 0,029 \\
\hline \multicolumn{7}{|l|}{ Edad } \\
\hline 18-20 años & $40(74,1)$ & $14(25,9)$ & Referencia & & Referencia & \\
\hline 21-26 años & $38(60,3)$ & $25(39,7)$ & $1,53(0,89-2,64)$ & 0,127 & $1,75(1,14-2,70)$ & 0,011 \\
\hline \multicolumn{7}{|l|}{ Tipo de Universidad } \\
\hline Universidad pública & $10(71,4)$ & $4(28,6)$ & Referencia & & -- & -- \\
\hline Universidad privada & $68(66,0)$ & $35(34,0)$ & $1,19(0,49-2,85)$ & 0,689 & -- & -- \\
\hline \multicolumn{7}{|l|}{ Localización de la Universidad } \\
\hline Universidad fuera de Lima & $20(54,1)$ & $17(45,9)$ & Referencia & & Referencia & -- \\
\hline Universidad de Lima & $58(72,5)$ & $22(27,5)$ & $0,60(0,36-0,99)$ & 0,045 & $0,79(0,51-1,22)$ & 0,293 \\
\hline \multicolumn{7}{|l|}{ Año de estudios } \\
\hline 1er-3er Año & $43(68,3)$ & $20(31,7)$ & Referencia & & -- & -- \\
\hline 4to-7mo Año & $35(64,8)$ & $19(35,2)$ & $1,10(0,22-0,45)$ & 0,695 & -- & -- \\
\hline \multicolumn{7}{|l|}{ Años activo en IFMSA } \\
\hline Al menos 1 año & $51(63,8)$ & $29(36,3)$ & Referencia & & -- & -- \\
\hline Más de un año & $27(73,0)$ & $10(27,0)$ & $0,75(0,41-1,37)$ & 0,343 & -- & -- \\
\hline \multicolumn{7}{|l|}{ Comité de IFMSA } \\
\hline SCOPH/SCORA/SCORP & $50(80,6)$ & $12(19,4)$ & Referencia & & Referencia & \\
\hline Otros & $20(50,0)$ & $20(50,0)$ & $2,58(1,42-4,69)$ & 0,002 & $2,18(1,25-3,80)$ & 0,006 \\
\hline Ninguno & $8(53,3)$ & $7(46,7)$ & $2,41(1,14-5,08)$ & 0,021 & $1,70(0,85-3,43)$ & 0,135 \\
\hline \multicolumn{7}{|l|}{ Religión } \\
\hline Católico & $60(64,5)$ & $33(35,5)$ & Referencia & & -- & -- \\
\hline Ateo & $5(83,3)$ & $1(16,7)$ & $0,47(0,08-2,89)$ & 0,415 & -- & -- \\
\hline Otros & $13(72,2)$ & $5(27,8)$ & $0,78(0,35-1,73)$ & 0,547 & -- & -- \\
\hline \multicolumn{7}{|l|}{ Familiar o amigo gay/lesbiana } \\
\hline No tiene o no sabe & $17(43,6)$ & $22(56,4)$ & Referencia & & Referencia & \\
\hline Sí tiene & $61(78,2)$ & $17(21,8)$ & $0,39(0,23-0,64)$ & $<0,001$ & $0,38(0,24-0,61)$ & $<0,001$ \\
\hline \multicolumn{7}{|l|}{ Compromiso religioso } \\
\hline Poco/ muy poco religioso(a) & $33(78,6)$ & $9(21,4)$ & Referencia & & Referencia & \\
\hline Algo/ muy religioso(a) & $45(60,0)$ & $30(40,0)$ & $1,87(0,98-3,56)$ & 0,058 & $1,89(1,07-3,37)$ & 0,030 \\
\hline
\end{tabular}

* Se ajustó por todas las variables mostradas en el modelo ajustado, que fueron elegidas debido a tener un p<0,20 en los análisis crudos. ATLG: Attitudes Toward Lesbians and Gay Men Scale; RP: razón de prevalencias; IC95\%: intervalo de confianza al 95\%; IFMSA: International Federation of Medical Students Associations; SCOPH: Standing Committee on Public Health; SCORA: Standing Committee on Sexual and Reproductive Health including HIV/AIDS; SCORP: Standing Committee on Human Rights and Peace.

\section{Otras variables}

Otras variables colectadas fueron: edad, sexo, universidad, año en curso (la carrera de medicina humana se desarrolla en 7 años promedio, esta variable fue categorizada en dos grupos uno que comprendía a estudiantes del primer al tercer año y la segunda del cuarto al séptimo año, la división se realizó de esa forma ya que a partir del cuarto año en la mayoría de universidades empiezan los cursos y rotaciones en el área clínica), comité de IFMSA al que pertenecía (SCOPH, SCORA, SCORP, SCOME, SCOPE, o SCORE), y religión que profesa.

\section{Análisis de los datos}

Los datos se digitaron por dos personas por separado y posteriormente fueron cruzados por una tercera para evitar 


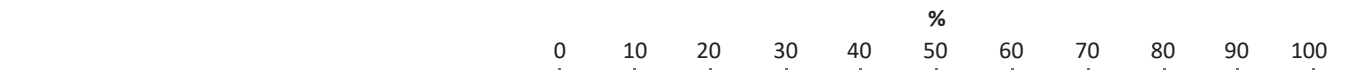

2) Pienso que los hombres homosexuales son repugnantes

3) A los hombres homosexuales NO debería permitírseles enseñar en los colegios

4) La homosexualidad masculina es una perversión

6) Si un hombre tiene sentimientos homosexuales, debería hacer todo lo posible para superarlos

8) El sexo entre dos hombres NO es natural

9) La idea del matrimonio homosexual me parece ridícula

1) A las parejas de hombres homosexuales debería permitírsele adoptar hijos como a las parejas heterosexuales *

5) La homosexualidad masculina es una expresión natural de la sexualidad masculina

7) Si supiera que mi hijo es homosexual yo NO estaría deprimido/a *

10) La homosexualidad masculina es un tipo diferente de opción de vida que NO debería ser condenada

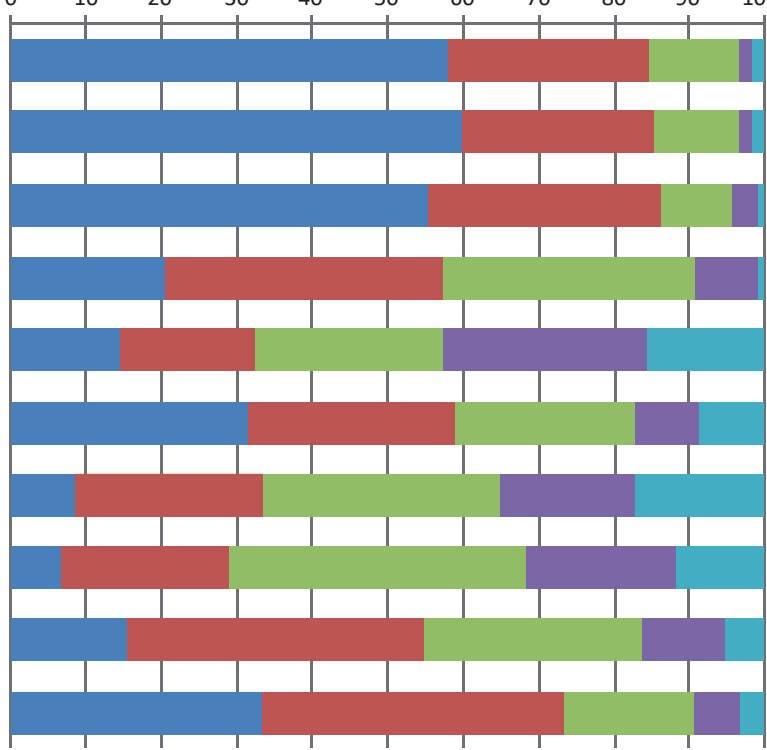

- Totalmente en desacuerdo $\quad$ En desacuerdo $\quad$ Ni en acuerdo ni en desacuerdo $\quad$ De acuerdo $\quad$ Totalmente de acuerdo

* Estos ítems representan actitudes de aceptación; los demás ítems representan actitudes negativas.

Figura 1. Actitud frente a homosexuales hombres, en un grupo de estudiantes de medicina peruanos: frecuencia de respuestas.

errores. Los análisis se realizaron en el programa estadístico STATA v.12. Para el análisis descriptivo, se calcularon medidas de tendencia central, medidas de dispersión, frecuencias absolutas y frecuencias relativas. Además, se evaluaron los factores asociados a haber tenido un puntaje mayor a 40 en la escala ATLG. Para lo cual se usó la regresión de Poisson con varianzas robustas, de donde se calcularon las razones de prevalencias (RP) crudas y ajustadas, con su respectivo intervalo de confianza al 95\% (IC95\%). La regresión ajustada incluyó todas las variables que presentaron un $\mathrm{p}<0,20$ en las regresiones crudas.

\section{Aspectos éticos}

La encuesta contó con un consentimiento informado. Además, se aseguró la voluntariedad, el anonimato y la confidencialidad de los participantes.

\section{RESULTADOS}

De los 150 asistentes a la AGO, 117 aceptaron participar en el estudio y devolvieron la encuesta respondiendo correctamente a todas las preguntas (aceptación del $78 \%$ ). El sexo femenino correspondió al $62,4 \%$, la edad promedio fue de $20,9 \pm 1,6$ años y el $79,5 \%$ fueron católicos. El 31,6\% participaba activamente en IFMSA por más de un año. Las características sociodemográficas se presentan en la Tabla 1.

En cuanto a las actitudes de rechazo que forman parte de la escala ATLG para hombres homosexuales, se encontró que más de la mitad de los encuestados estuvieron de acuerdo o muy de acuerdo en que el sexo entre dos hombres no era natural. En cuanto a las actitudes positivas, menos del $40 \%$ de los encuestados estuvieron de acuerdo o muy de acuerdo con que "a las parejas de hombres homosexuales debería permitírseles adoptar hijos como a las parejas heterosexuales", "la homosexualidad masculina es una expresión natural de la sexualidad masculina", "si supiera que mi hijo es homosexual yo NO estaría deprimido/a", y "la homosexualidad masculina es un tipo diferente de opción de vida que NO debería ser condenada"; como se muestra en la Figura 1.

Al evaluar los puntajes, se encontró que 10 personas $(8,5 \%)$ marcaron estar muy en desacuerdo con todas las actitudes negativas, y muy de acuerdo con todas las actitudes positivas. El puntaje sigue una distribución asimétrica, con una cola a la derecha, como se observa en la Figura 2.

Treinta y nueve participantes $(33,3 \%)$ tuvieron más de 40 puntos, lo cual calificamos como una actitud negativa frente a los homosexuales varones. Al evaluar los factores asociados a este puntaje, se encontró que, en el modelo ajustado, tener un mayor nivel de compromiso religioso (RP: 1,89; IC95\%: 1,07-3,37), tener una edad mayor (RP: 1,$75 ;$ IC $95 \%: 1,14-2,70$ ), ser de un comité distinto a SCOPH/SCOPA/SCORP (RP: 2,18; IC95\%: 1,25-3,80). Asimismo, ser mujer y tener algún amigo o familiar gay/lesbiana fueron factores que disminuyeron la probabilidad de tener actitudes negativas hacia hombres homosexuales (Tabla 2). 


\section{DISCUSIÓN}

El presente estudio ha sido realizado en una población de estudiantes de medicina que participan en la IFMSA-Perú. Esta asociación tiene una posición de respeto y aceptación ante las diferentes orientaciones sexuales, por lo que probablemente aquellos estudiantes con actitudes negativas frente a los homosexuales estarían menos propensos a unirse a esta asociación. En consecuencia, nuestros resultados subestimarían el nivel de actitudes negativas de los estudiantes de medicina humana del Perú. No obstante, no tenemos razones para pensar que la asociación entre compromiso religioso y actitudes negativas esté alterada.

El 42,7\% de los encuestados estuvieron de acuerdo o totalmente de acuerdo con que el sexo entre dos hombres no era natural. Esta percepción se debería al escaso conocimiento de la población sobre las causas de la homosexualidad, creyendo que ésta se debe a alteraciones biológicas, mentales, hormonales, o experiencias con gran impacto psicológico como el abuso sexual ${ }^{[27]} ;$ a pesar de que actualmente se han encontrado indicios sólidos de que la homosexualidad tendría un componente genético ${ }^{[28]}$.

El 35,0\% de los encuestados está en desacuerdo o totalmente en desacuerdo con que las parejas homosexuales deberían poder adoptar hijos. Esto es considerado por los sectores conservadores como un atentado contra los valores familiares, que perjudicaría la salud mental y la moral de los niños ${ }^{[29]}$. Sin embargo, estudios recientes revelan que no habría diferencias significativas en la comunicación entre los padres y niños ${ }^{[30]}$, ni en el funcionamiento emocional y cognitivo de los niños con padres homosexuales respecto a los adoptados por parejas

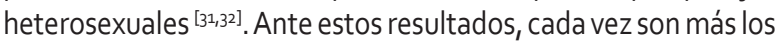
países en los que se permite la adopción conjunta por personas homosexuales ${ }^{[33]}$. Sin embargo, en ciertos contextos estos niños pueden sentirse discriminados por su entorno ${ }^{[34]}$.

El grado de compromiso religioso, más no la religión, mostró asociación con las actitudes negativas frente a los homosexuales. Esto coincide con otros estudios realizados en Serbia ${ }^{[35]}$, Estados Unidos ${ }^{[36,37]}$ e Inglaterra ${ }^{[38]}$ y 79 ciudades a nivel mundial ${ }^{[39]}$. Ciertas religiones parecen estar asociadas al rechazo moral de la homosexualidad, pero tener una escasa asociación al rechazo a los homosexuales como grupo; en tanto que en otras religiones están asociadas al rechazo de los homosexuales como grupo ${ }^{[40]}$.

La asociación entre compromiso religioso y rechazo a los homosexuales podría explicarse debido a una falta de información en torno a la homosexualidad y la creencia de ideas erróneas o prejuicios que no se ajustan a la realidad, creando una imagen tergiversada de estas personas ${ }^{[4]]}$. Sin embargo, se postula que la modernización está gestando un proceso de liberalización social que incluye cambios de actitudes hacia la homosexualidad ${ }^{[39]}$. En este contexto, existen comunidades religiosas, iglesias y organizaciones que tienen una mayor apertura y un mensaje de bienvenida a la comunidad LGTB en distintas religiones ${ }^{[42]}$.

Se encontró que las mujeres tuvieron menos actitudes negativas frente a los hombres homosexuales. Esto coincide con otros estudios realizados en Chile ${ }^{[26]}$, México ${ }^{[16]}$ y Reino Unido ${ }^{[43]}$. Esto se debería a que los varones, en sociedades predominantemente machistas como la peruana, rechazarían la conducta homosexual al relacionarla con debilidad, asociación que atentaría contra su identidad masculina dominante ${ }^{[44]}$.

En nuestra población, los encuestados con una edad de 21 años o más tuvieron mayor frecuencia de actitudes desfavorables, resultando que concuerda con un estudio realizado en Portugal donde los

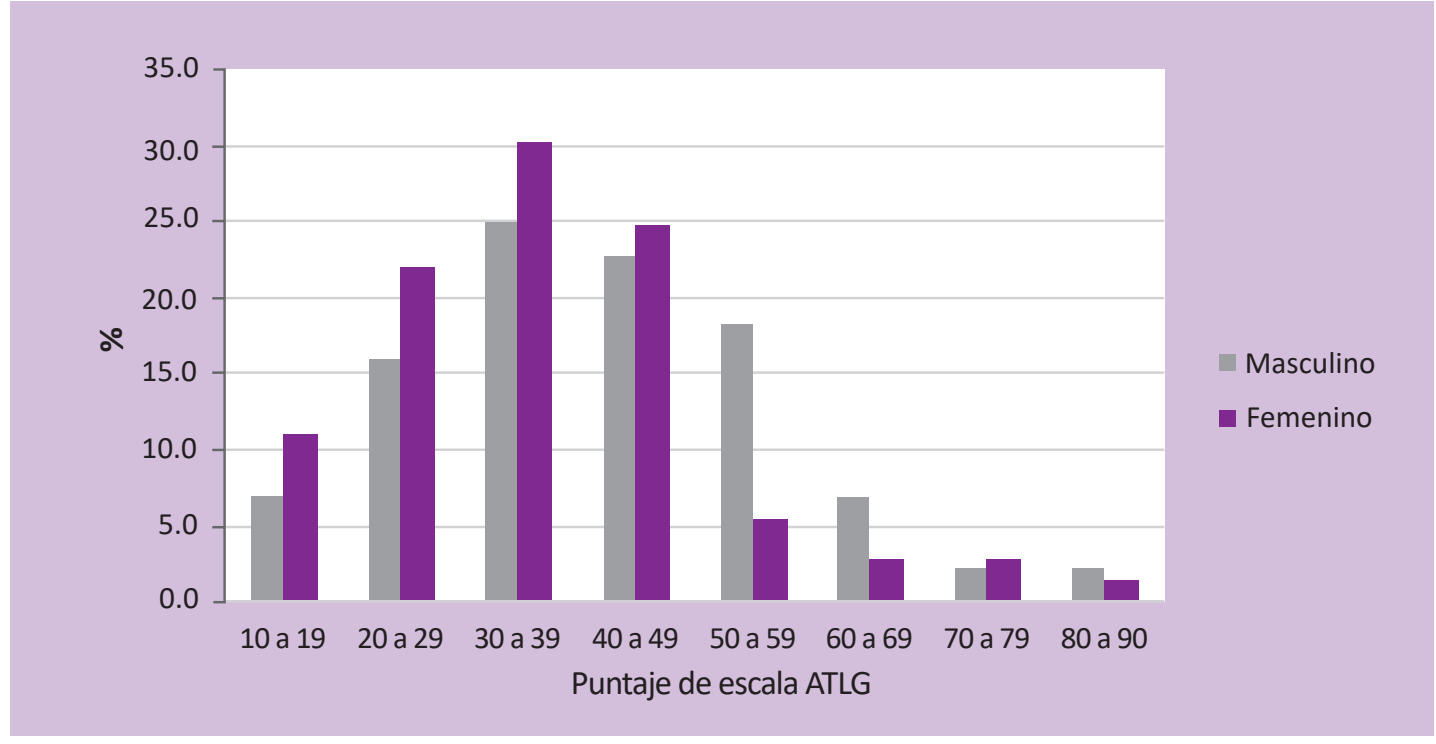

ATLG: Attitudes Toward Lesbians and Gay Men Scale

Figura 2. Puntajes obtenidos en una dimensión de la escala ATLG para evaluar la actitud frente a homosexuales hombres, en un grupo de estudiantes de medicina peruanos 
participantes mayores a 23 años mostraron una actitud desfavorable hacia los homosexuales ${ }^{[11]}$, pero diverge de otro estudio ejecutado en el Reino Unido que no encontró diferencia significativa entre las edades ${ }^{[43]}$. Nuestros resultados pueden deberse a que los miembros más jóvenes -menores de 21 años- tendrían un mayor acceso a educación formal e información sobre homosexualidad ${ }^{[45]}$.

Los participantes que pertenecieron a SCOPH/SCORA/SCORP tuvieron mejores actitudes que los demás. Estos comités tienen como objetivos afines educar y abogar por políticas de salud sexual y reproductiva y derechos humanos ${ }^{[46]}$, por lo cual es de esperar que sus miembros tengan una mayor educación en estos temas ${ }^{[4]]}$, lo cual reduce los prejuicios ante la homosexualidad. Sin embargo, también es posible que quienes tengan mejores actitudes hacia la homosexualidad presenten más interés en unirse a estos comités. En todo caso, la direccionalidad de esta asociación debería ser estudiada en futuros estudios.

Tener un familiar o un amigo gay estuvo asociado a mejores actitudes frente a los varones homosexuales, resultado similar

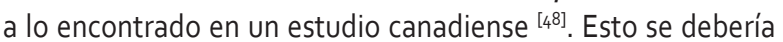
a que el trato continúo con personas homosexuales ayudaría a romper ciertos prejuicios sobre la homosexualidad. Este hallazgo reforzaría la idea de que visibilizar la homosexualidad ayudaría a incrementar su aceptación en los círculos cercanos.

Ante este panorama se pueden plantear soluciones que involucren a líderes políticos, cívicos y religiosos que influyan en la opinión pública y el establecimiento de políticas sociales que eviten la persecución contra la comunidad LGTB ${ }^{[42]}$. Además, resulta importante generar un ambiente tolerante para las personas homosexuales en las iglesias con una postura de bienvenida, sobre todo para aquellas quienes viven un conflicto interno entre su religiosidad y su homosexualidad, lo cual permitiría una participación más activa en la iglesia y consolidaría su compromiso religioso y espiritualidad [49].

En el entorno universitario se debería buscar que los estudiantes concilien sus creencias religiosas con actitudes positivas frente a homosexuales, quienes estarán entre sus futuros pacientes. Para ello, se podría desarrollar temas de orientación sexual dentro del plan de estudios [49], invitar a ponentes homosexuales ${ }^{[50]}$, brindar charlas de políticas sobre derechos humanos y contra la discriminación ${ }^{\left[5^{1]}\right.}$ y evaluar en qué medida estas y otras estrategias promueven un cambio de percepción hacia los homosexuales ${ }^{[43]}$. En nuestro estudio, se pudo observar que las personas ateas mostraban mejores actitudes hacía los hombres homosexuales; no obstante, el pequeño tamaño muestral -6 personas- explicaría su no significancia estadística. Se requerirán futuros estudios que evalúen a esta población en particular.

Nuestro estudio presenta limitaciones. Al analizar una submuestra de estudiantes de medicina peruanos, es probable que hayamos subestimando las actitudes negativas y haría que los resultados reportados sean aún más alarmantes. En cuanto a la religiosidad, ésta podría haber sido medida con otros instrumentos, como la escala de religiosidad de Duke (DUREL, por sus siglas en inglés), que brindarían mejor detalle de la característica a evaluar.
Otra limitación es que se aplicó únicamente la primera parte de la escala ATLG, limitando los resultados a las actitudes frente a los homosexuales hombres. Además, dicha escala incluye una pregunta que utiliza el término "opción de vida" en relación a la homosexualidad, lo que podría confundir a los encuestados ya que en la actualidad se acepta que la homosexualidad no es una opción, sino una orientación; por tanto, sería necesario realizar una adaptación cultural y validación local del instrumento.

A pesar de lo mencionado, este es el primer estudio que evalúa factores asociados a las actitudes frente a homosexuales en estudiantes de medicina, y los resultados muestran la vigencia de este problema en nuestro país, asi como que existen grupos que podrían estar en mayor riesgo de presentar actitudes negativas frente a los homosexuales. Sirve también como referencia para que las universidades y otras instituciones interesadas desarrollen e implementen futuros estudios con mayor número de población.

En conclusión, encontramos que los estudiantes encuestados tienen actitudes negativas frente a los hombres homosexuales. Aquellos con mayor nivel de compromiso religioso presentaron una mayor actitud negativa. Existe la necesidad de intervenir en los estudiantes de medicina humana con el objetivo de conciliar sus creencias religiosas con la formación de médicos preparados para atender y aconsejar asertivamente a las personas homosexuales.

\section{REFERENCIAS BIBLIOGRÁFICAS}

1. Cáceres C, Talavera V, Reynoso R. Diversidad sexual, salud y ciudadanía. Rev Peru Med Exp Salud Publica. 2013;30(4):698-704

2. Ardila R. Terapia afirmativa para homosexuales y lesbianas. Rev Colomb Psiquiatr. 2007;31(1):67-77.

3. American Psychiatric Association. Diagnostic and statistical manual of mental disorders. 2nd ed. Washington, DC: APA; 1968.

4. Campo-Arias A, Herazo E, CogolloZ. Homophobia among nursing students. Rev Esc Enferm USP. 2010;44(3):839-43.

5. Albuquerque GA, de Lima GC, da Silva QG, Alves MJH, Belém JM, dos Santos FFW, et al. Access to health services by lesbian, gay, bisexual, and transgender persons: systematic literature review. BMC Int Health Hum Rights. 2016;16:2. doi: 10.1186/s12914-015-0072-9.

6. Hernández-Rosete D. La otra migración. Historias de discriminación de una persona con VIH en México. Salud Ment. 2008;31(4):253-60.

7. Movimiento de Integración y Liberación Homosexual. X Informe Anual de Derechos Humanos de la Diversidad sexual en Chile. Santiago, Chile: Movilh; 2012.

8. Barrientos-Delgado J, Cárdenas-Castro M, Gómez-Ojeda F. Características sociodemográficas, bienestar subjetivo y homofobia en una muestra de hombres gay en tres ciudades chilenas. Cad Saúde Pública. 2015;31(4):767-76.

9. Piña JM, Aguayo HB. Homofobia en estudiantes universitarios de México. Región y sociedad. 2015;27(64):5-35.

10. Kirby BJ, Michaelson C. Comparative Morality Judgments About Lesbians and Gay Men Teaching and Adopting Children. J Homosexual. 2015;62(1):33-50.

11. Gomes IS, Serôdio RG. A homofobia perspetivada à luz da abordagem da identidade social: Níveis de autodefinição identitária e atitude em relação a pessoas homossexuais. Anál Psicológica. 2014;32(2):215-30. 
12. Campo-Arias A, Oviedo HC, Herazo E. Correlación entre homofobia y racismo en estudiantes de medicina. Psicol Caribe. 2014;31(1):25-37.

13. Hausmann LR, Hannon MJ, Kresevic DM, Hanusa BH, Kwoh CK, Ibrahim SA. Impact of perceived discrimination in health care on patient-provider communication. Med Care. 2011;49(7):626-33

14. Campo-Arias A, Herazo E. Homofobia en estudiantes de medicina: una revisión de los diez últimos años. Medunab. 2008;11(2):120-3.

15. Banwari G, Mistry K, Soni A, Parikh N, Gandhi H. Medical students and interns' knowledge about and attitude towards homosexuality. J Postgrad Med. 2015;61(2):95-100.

16. de la Rubia J, Valle A, Martínez E. Evaluación del rechazo hacia la homosexualidad en estudiantes de medicina y psicología con base en tres escalas conceptualmente afines. Psicol Caribe. 2013;30(3):526-50.

17. Cáceres VS. Actitudes sobre el aborto, matrimonio homosexual y otros aspectos socio sexuales en estudiantes del sexto año de medicina humana en una universidad estatal. Rev Cuerpo Med HNAAA. $2014 ; 7(2): 6-10$

18. de la Rubia J, Valle A. Escala de actitudes hacia lesbianas y hombres homosexuales en México 1. Estructura factorial y consistencia interna. Nova Scientia. 2011;3(6):139-57.

19. Moreno A, Herazo E, Oviedo H, Campo-Arias A. Measuring homonegativity: psychometric analysis of Herek's attitudes toward lesbians and gay men scale (ATLG) in Colombia, South America. J Homosexual. 2015;62(7):924-35.

20. Cárdenas M, Barrientos J. The attitudes toward lesbians and gay men scale (ATLG): adaptation and testing the reliability and validity in Chile. J Sex Res. 2008;45(2):140-9.

21. Delgado JEB, Castro JMC. Adaptación y validación de la escala Likert de actitudes de heterosexuales hacia homosexuales (HATH) en una muestra de estudiantes universitarios chilenos. Sex, Salud Soc Rev Latinoamer. 2010;(5):30-49.

22. Curlin FA, Lantos JD, Roach CJ, Sellergren SA, Chin MH. Religious characteristics of US physicians. J Gen Intern Med. 2005;20(7):629-34.

23. Curlin FA, Odell SV, Lawrence RE, Chin MH, Lantos JD, Meador KG, et al. The relationship between psychiatry and religion among US physicians. Psychiatr Serv. 2007;58(9):1193-8.

24. Ortiz-Hernández L, García M. Efectos de la violencia y la discriminación en la salud mental de bisexuales, lesbianas y homosexuales de la Ciudad de México. Cad Saúde Pública. 2005;21(3):913-25.

25. Moral de la Rubia J. Escala de Actitudes hacia Lesbianas y Hombres Homosexuales (ATLG) 2: Distribución y evidencias de validez. Nova scientia. 2012;4(7):153-71.

26. Moral de la Rubia J. Dimensionalidad, consistencia interna y distribución de la escala homonegatividad internalizada en estudiantes mexicanos de ciencias de la salud. Acta de investigación psicol. 2013;3(1):986-1004

27. Beirute-Herrera J, Angulo-Castro V, Fallas-Gamboa K, PradoCalderón JE. Efecto del asco sobre las actitudes hacia hombres homosexuales: evidencias de cognición corporalizada en una muestra costarricense. Rev Wímb Lu. 2014;9(2):7-21.

28. Cárdenas M, Barrientos J. Actitudes explícitas e implícitas hacia los hombres homosexuales en una muestra de estudiantes universitarios en Chile. Psykhe. 2008;17(2):17-25

29. Drescher J. Out of DSM: Depathologizing Homosexuality. Behav Sci (Basel). 2015;5(4):565-75.

30. Camperio A, Battaglia U, Zanzotto G. Human Homosexuality: A Paradigmatic Arena for Sexually Antagonistic Selection? Cold Spring Harb Perspect Biol. 2015;7(4):a017657.

31. Briggs L. Somebody's children: The politics of transnational and transracial adoption. Durham, NC: Duke University Press; 2012.
32. Golombok S, Mellish L, Jennings $S$, Casey P, Tasker F, Lamb ME. Adoptive gay father families: Parent-child relationships and children's psychological adjustment. Child Dev. 2014;85(2):456-68.

33. Goldberg AE, Smith JZ. Predictors of school engagement among same-sex and heterosexual adoptive parents of Kindergarteners. J Sch Psychol. 2014;52(5):463-78.

34. Anderssen N, Amlie C, Ytterøy EA. Outcomes for children with lesbian or gay parents. A review of studies from 1978 to 2000 . Scand J Psychol. 2002;43(4):335-51.

35. Dunjić-Kostić B, Pantović M, Vuković V, Randjelović D, TotićPoznanović S, Damjanović A, et al. Knowledge: A possible tool in shaping medical professionals'attitudes towards homosexuality. Psychiatr Danub. 2012;24(2):143-51.

36. Adamczyk A, Pitt C. Shaping attitudes about homosexuality: the role of religion and cultural context. Soc Sci Res. 2009;38(2):338-51.

37. Adamczyk A, Boyd KA, Hayes BE. Place matters: Contextualizing the roles of religion and race for understanding Americans' attitudes about homosexuality. Soc Sci Res. 2016;57:1-16.

38. Parker ADB. Attitudes of British medical students towards male homosexuality. Sexual and Relationship Therapy. 2000;15(2):141-9.

39. Jackle S, Wenzelburger G. Religion, Religiosity, and the Attitudes Toward Homosexuality-A Multilevel Analysis of 79 Countries. J Homosex. 2014;62(2):207-41.

40. Doebler S. Relationships between Religion and Two Forms of Homonegativity in Europe-A Multilevel Analysis of Effects of Believing, Belonging and Religious Practice. PloS one. 2015;10(8):e0133538.

41. Saraç L. Relationships between religiosity level and attitudes toward lesbians and gay men among Turkish university students. J Homosex. 2015;62(4):481-94.

42. Liboro RM. Community-level interventions for reconciling conflicting religious and sexual domains in identity incongruity. J Relig Health. 2015;54(4):1206-20.

43. Ellis $S$, Kitzinger C, Wilkinson S. Attitudes towards lesbians and gay men and support for lesbian and gay human rights among psychology students. J Homosex. 2003;44(1):121-38

44. Verduzco IL, Sánchez TER. La homofobia y su relación con la masculinidad hegemónica en México. Revista Puertorriqueña de Psicología. 2011;22:101-21.

45. Steffens MC, Wagner C. Attitudes toward lesbians, gay men, bisexual women, and bisexual men in Germany. J Sex Res. 2004; $41(2): 137-49$.

46. International Federation of Medical Students' Associations [Internet]. Amsterdam: IFMSA;C2017 [citedo el 5 de enero de 2016]. Disponible en: https://ifmsa.org/

47. Ross MW, Nyoni J, Larsson M, Mbwambo J, Agardh A, Kashiha J, et al. Health care in a homophobic climate: the SPEND model for providing sexual health services to men who have sex with men where their health and human rights are compromised. Glob Health Action. 2015 Mar 17;8:260g6.

48. Rye BJ, Meaney GJ. Impact of a homonegativity awareness workshop on attitudes toward homosexuality. J Homosex. 2009;56(1):31-55.

49. Subhi N, Geelan D, McMahon M, Jusoff K, Mohamad S, Sarnon $\mathrm{N}$, et al. A better understanding of the potential conflict between Christianity and homosexuality. World Appl Sci J. 2011;12:13-9.

50. Buhrke RA. Female student perspectives on training in lesbian and gay issues. Counseling Psychologist. 1989;17(4):629-36.

51. Berkman CS, Zinberg G. Homophobia and heterosexism in social workers. Soc Work. 1997;42(4):319-32. 